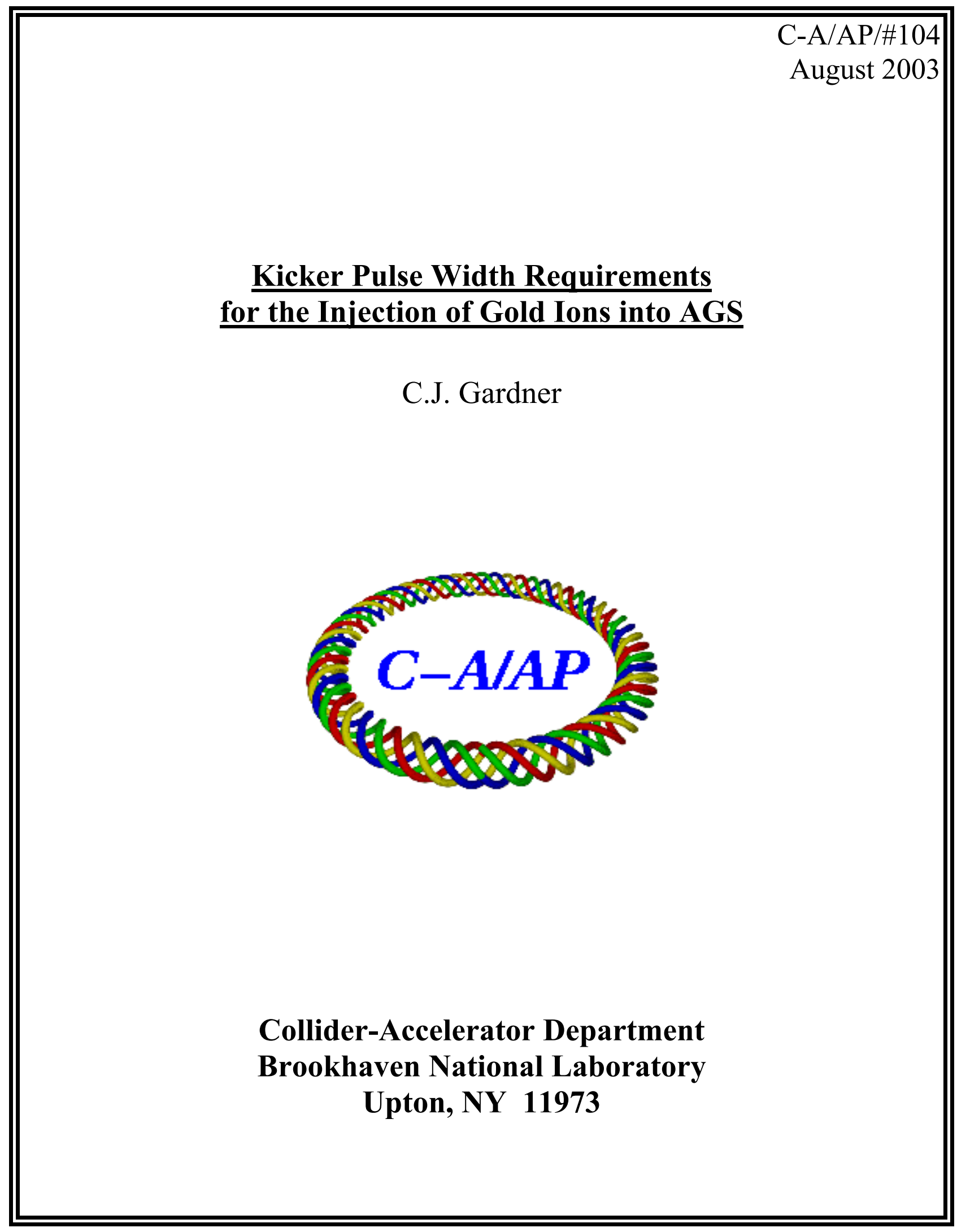




\title{
Kicker Pulse Width Requirements for the Injection of Gold Ions into AGS
}

\author{
C.J. Gardner
}

July 29, 2003

A new scheme proposed by J.M. Brennan for the acceleration of gold ions in Booster and AGS, requires a shorter pulse width for the injection kicker located in the A5 straight section of the AGS. We review here the pulse width requirements for the "old" scheme used for the past several years and then examine the requirements for the new scheme.

\section{General Formulae for Required Pulse Width}

We begin by writing down some general formulae for the required pulse width. Let us assume that $M$ loads of $N$ bunches are transfered from Booster and injected into stationary RF buckets in AGS. We assume that $M \times N$ bunches fill all available buckets. Let $T$ be the center-to-center spacing (in time) of the bunches of a load coming into AGS, and let $W_{B}$ be the incoming bunch width. The field in the A5 kicker takes rise time $T_{R}$ to go from zero to the value $A$ required to put the incoming bunches onto the AGS EO (Equilibrium Orbit). In order to put all $N$ bunches of a load onto the EO, the field must be held at the value $A$ for time

$$
W=(N-1) T+W_{B} .
$$

We call this the required width of the kicker pulse. After injection of the $N$ bunches, the field in the kicker returns to zero in fall time $T_{F}$. For each transfer of $N$ bunches, the total time that the field is nonzero is then

$$
\widehat{W}=T_{R}+W+T_{F} .
$$

We call this the total width of the pulse. 
Now, in the BTA (Booster-To-AGS) transfer line, the bunches pass through a stripping foil. Although the spacing of the bunches in time is unaffected by passage through the foil, the bunches emerge from the foil with reduced velocity. Assuming the length of the AGS EO does not change, the revolution frequency of the bunches in AGS is then reduced and the center-to-center spacing of the stationary RF buckets into which the bunches are injected increases from $T$ to $T+d t$. This subtle mismatch between bunch and bucket spacing, first noticed by Brennan, causes filamentation of the incoming bunches in the RF buckets. Filamentation also occurs if the shape of the bucket is not matched to that of the incoming bunch. Let $W_{F}$ be the final width of the bunch after filamentation in an RF bucket. Then the time gap available for the last transfer (i.e. for the $M$ th transfer) to AGS is

$$
T_{G}=(N+1)(T+d t)-W_{F} .
$$

The total width of the kicker pulse, $\widehat{W}$, must be less than $T_{G}$. Thus

$$
T_{R}+W+T_{F}<T_{G}
$$

and

$$
T_{R}+T_{F}<T_{G}-W
$$

where

$$
T_{G}-W=2 T+(N+1) d t-\left(W_{B}+W_{F}\right) .
$$

We require further that the $N$ incoming bunches of the final transfer be centered in the available gap. We then must have

$$
T_{R}<\left(T_{G}-W\right) / 2, \quad T_{F}<\left(T_{G}-W\right) / 2 .
$$

In the following two sections we apply these formulae to the old and new acceleration schemes.

\section{The Old Scheme}

The old scheme used for the past several years is described in detail in Ref. [1]. Four loads of six bunches are transfered from Booster to AGS and these fill $24 \mathrm{RF}$ buckets on the AGS injection porch. The longitudinal parameters are summarized in Table 1. Here $\epsilon$ is the longitudinal emittance of a single bunch (the unit $\mathrm{eV}-\mathrm{s} / \mathrm{n}$ means $\mathrm{eV}$-s per nucleon), $\Delta t$ 
and $\Delta E$ are the bunch width and half-height respectively, $h f$ is the $\mathrm{RF}$ frequency, and $1 /(h f)$ is the bunch spacing. The harmonic numbers are $h=6$ and $h=24$ in Booster and AGS respectively. The numbers in the table have been obtained from measurements and from the program "bbat".

Table 1: Longitudinal Parameters for Gold Ions (Old Scheme)

\begin{tabular}{|c|c|c|c|c|}
\hline Parameter & $\begin{array}{c}\text { Booster } \\
\text { Extraction }\end{array}$ & $\begin{array}{c}\text { After } \\
\text { BTA Foil }\end{array}$ & $\begin{array}{c}\text { After AGS } \\
\text { Filamentation }\end{array}$ & Unit \\
\hline$\epsilon$ & $0.045 / 6$ & $4 \times 0.045 / 6$ & $6 \times 0.045 / 6$ & $\mathrm{eV}-\mathrm{s} / \mathrm{n}$ \\
\hline$\Delta t$ & 48 & 48 & 76 & $\mathrm{~ns}$ \\
$\Delta E$ & 20 & 76 & 76 & $\mathrm{MeV}$ \\
\hline$h f$ & 3.848719 & 3.848719 & 3.775458 & $\mathrm{MHz}$ \\
$1 /(h f)$ & 259.827 & 259.827 & 264.868 & $\mathrm{~ns}$ \\
\hline
\end{tabular}

Because of energy straggling, the longitudinal emittance increases by a factor of four upon traversal of the BTA stripping foil. It increases by another $50 \%$ due to filamentation in the AGS RF bucket. This gives a total increase of a factor of six. As indicated in the table, the spacing of the bunches coming into AGS is $260 \mathrm{~ns}$ and this increases to $265 \mathrm{~ns}$ after filamentation in the harmonic 24 buckets. The incoming bunch width is 48 ns and this increases to $76 \mathrm{~ns}$ after filamentation. Thus, the parameter values to be used in the formulae of Section 1 are

$$
N=6, \quad T=260 \mathrm{~ns}, \quad d t=5 \mathrm{~ns}, \quad W_{B}=48 \mathrm{~ns}, \quad W_{F}=76 \mathrm{~ns}
$$

which give

$$
W=1348 \mathrm{~ns}, \quad T_{G}=1779 \mathrm{~ns}, \quad T_{R}<216 \mathrm{~ns}, \quad T_{F}<216 \mathrm{~ns} .
$$

The A5 kicker timing for the injection of $N=6$ bunches per transfer is illustrated in Figure 1. The mismatch between bunch and bucket spacing is illustrated in Figure 2. Note that the width and fall time given by (9) are just barely within the capability of the A5 kicker in the "Long Pulse" mode. Oscilliscope traces of the waveform for this mode are shown in Figure 3.

After injection, the 24 bunches are adiabatically debunched and rebunched into 4 bunches. Each of these bunches has an emittance of $6 \times 0.045=0.27$ eV-s per nucleon. 


\section{The New Scheme}

The new scheme proposed by J.M. Brennan for the next RHIC run still involves harmonic six in Booster and harmonic 24 on the AGS injection porch, but harmonic three is now introduced in Booster to merge the six bunches into three on a porch approximately one third of the way between injection and top magnetic field. After the six bunches have been merged into three $h=3$ buckets, they are "squeezed" into three adjacent $h=6$ buckets on the porch. The result is that the beam in one Booster load which used to occupy six $h=6$ buckets now occupies three adjacent $h=6$ buckets. This doubles the intensity per bunch in Booster, and, with eight transfers of three bunches into the 24 buckets on the AGS injection porch, doubles the intensity in AGS. As before, the 24 bunches are debunched and rebunched into four bunches. This gives a final single-bunch intensity in AGS twice that of the old scheme.

Along with the intensity increase comes an increase in longitudinal emittance. This is at least a factor of two simply because two bunches are merged into one in Booster. We assume an additional increase of $50 \%$ due to filamentation during the merging and squeezing processes. This gives a total factor of three increase in the single-bunch emittance in Booster. With new more uniform stripping foils in the BTA line, the energy straggling in the foil has been shown to be reduced significantly [2]. We assume that the increase in emittance due to energy straggling and due to filamentation in the AGS RF buckets is at most a factor of two. This gives a total increase in the single-bunch longitudinal emittance of a factor of six just as in the old scheme (but now with twice the intensity). Table 2 shows the expected longitudinal parameters.

Table 2: Longitudinal Parameters for Gold Ions (New Scheme)

\begin{tabular}{|c|c|c|c|c|}
\hline Parameter & $\begin{array}{c}\text { Booster } \\
\text { Extraction }\end{array}$ & $\begin{array}{c}\text { After } \\
\text { BTA Foil }\end{array}$ & $\begin{array}{c}\text { After AGS } \\
\text { Filamentation }\end{array}$ & Unit \\
\hline$\epsilon$ & $0.135 / 6$ & $0.216 / 6$ & $2 \times 0.135 / 6$ & $\mathrm{eV}-\mathrm{s} / \mathrm{n}$ \\
\hline$\Delta t$ & 86 & 86 & 96 & $\mathrm{~ns}$ \\
$\Delta E$ & 34 & 53 & 60 & $\mathrm{MeV}$ \\
\hline$h f$ & 3.848719 & 3.848719 & 3.775458 & $\mathrm{MHz}$ \\
$1 /(h f)$ & 259.827 & 259.827 & 264.868 & $\mathrm{~ns}$ \\
\hline
\end{tabular}


Here, as before, $\Delta t$ and $\Delta E$ have been calculated using the program "bbat".

As pointed out by Brennan, there are a couple of things other than the improved uniformity of the foil that help us out here. The total time spent by the beam in the foil is reduced simply because the total width of three bunches is less than that of six. This by itself helps reduce the energy straggling in the foil. The mismatch between the spacing of the incoming bunches and the AGS RF buckets is less for three bunches than it is for six. The factor here is $5 / 2$ and this helps reduce filamentation in the AGS buckets. Finally, because there is less energy straggling in the foil, there is sufficient voltage to match the AGS buckets to the incoming bunches. In the old scheme, the voltage required for matching was a factor of 2.6 higher than what is available and this caused significant filamentation.

From Table 2 we see that the parameter values to be used in the formulae of Section 1 are

$$
N=3, \quad T=260 \mathrm{~ns}, \quad d t=5 \mathrm{~ns}, \quad W_{B}=86 \mathrm{~ns}, \quad W_{F}=96 \mathrm{~ns}
$$

which give

$$
W=606 \mathrm{~ns}, \quad T_{G}=964 \mathrm{~ns}, \quad T_{R}<179 \mathrm{~ns}, \quad T_{F}<179 \mathrm{~ns} .
$$

The A5 kicker timing for the injection of $N=3$ bunches per transfer is illustrated in Figure 4. The mismatch between bunch and bucket spacing is illustrated in Figure 5. The oscilliscope traces in Figure $\mathbf{3}$ show that the A5 pulse in the "Short Pulse" mode is not long enough for the parameters given in (11). If the "Long Pulse" mode is used, then only 7 of the 8 possible transfers could be injected into AGS. The present plan is to go back to the original short pulse (used for the injection of protons some years ago) which is some $100 \mathrm{~ns}$ longer than the present short pulse. This original pulse is shown in Figures 6 and 7. Here we see that the flat top part of the pulse is nearly $600 \mathrm{~ns}$ long which is very close to what is required. The rise time is $100 \mathrm{~ns}$ and, with the "tailbite", the fall time is $200 \mathrm{~ns}$. Although the fall time is somewhat longer than desired, this is critical only at the end of the last transfer where some loss may occur on the neighboring bunch of the first transfer. 


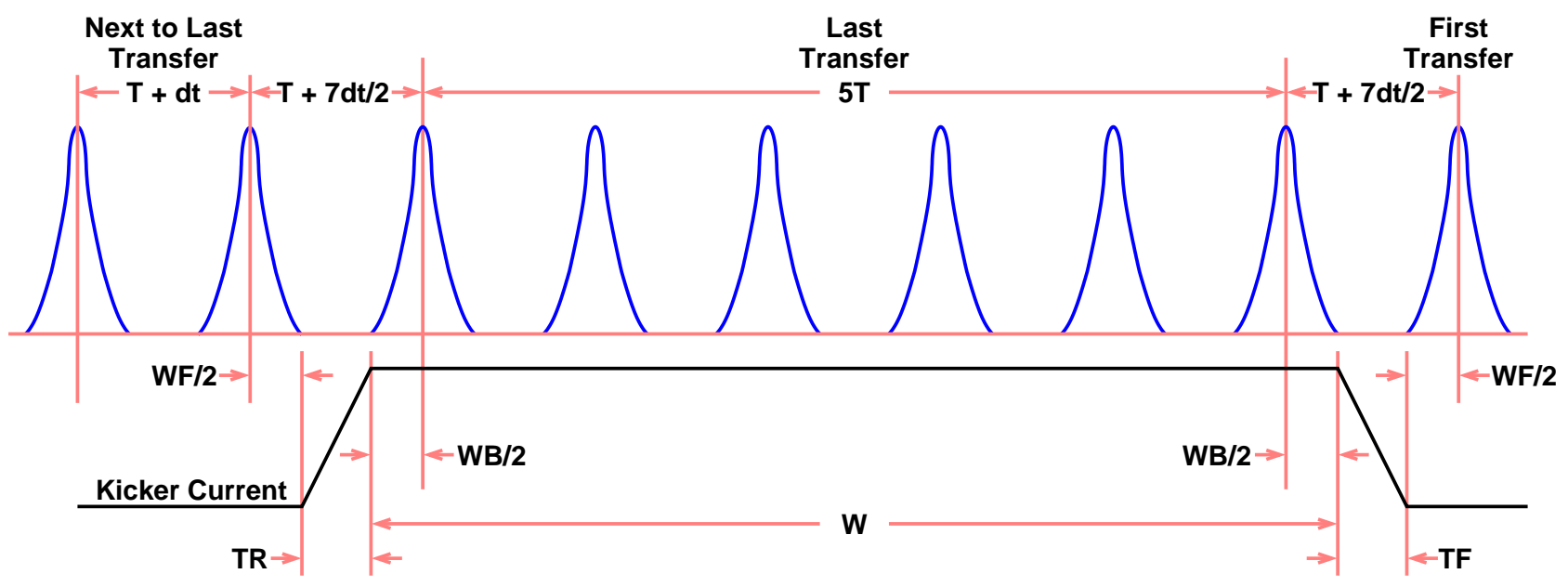

Figure 1: A5 Kicker timing for injection of $N=6$ bunches per transfer from Booster. Here $W=5 T+W_{B}$ and $T_{G}-W=2 T+7 d t-\left(W_{B}+W_{F}\right)$. For $T=260 \mathrm{~ns}, d t=5 \mathrm{~ns}, W_{B}=48 \mathrm{~ns}$, and $W_{F}=76 \mathrm{~ns}$, we have $W=1348$ $\mathrm{ns}, T_{G}=1779 \mathrm{~ns}, T_{R}<216 \mathrm{~ns}$, and $T_{F}<216 \mathrm{~ns}$.

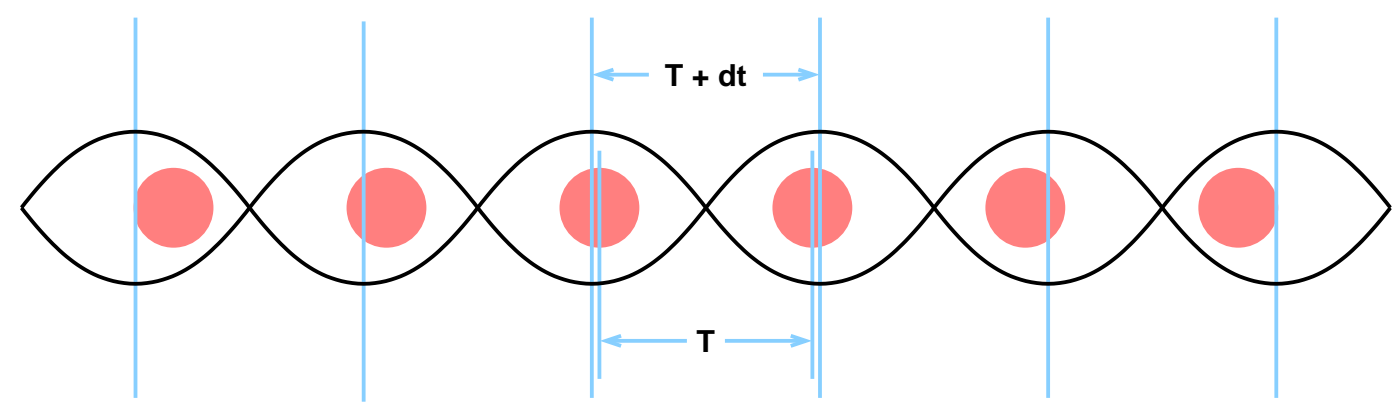

Figure 2: Phase mismatch between incoming bunches and RF buckets with six bunches per transfer. Here $T=260 \mathrm{~ns}$ and $d t=5 \mathrm{~ns}$. The displacement from center of bucket to center of bunch is $5 d t / 2$ in the outermost buckets. 


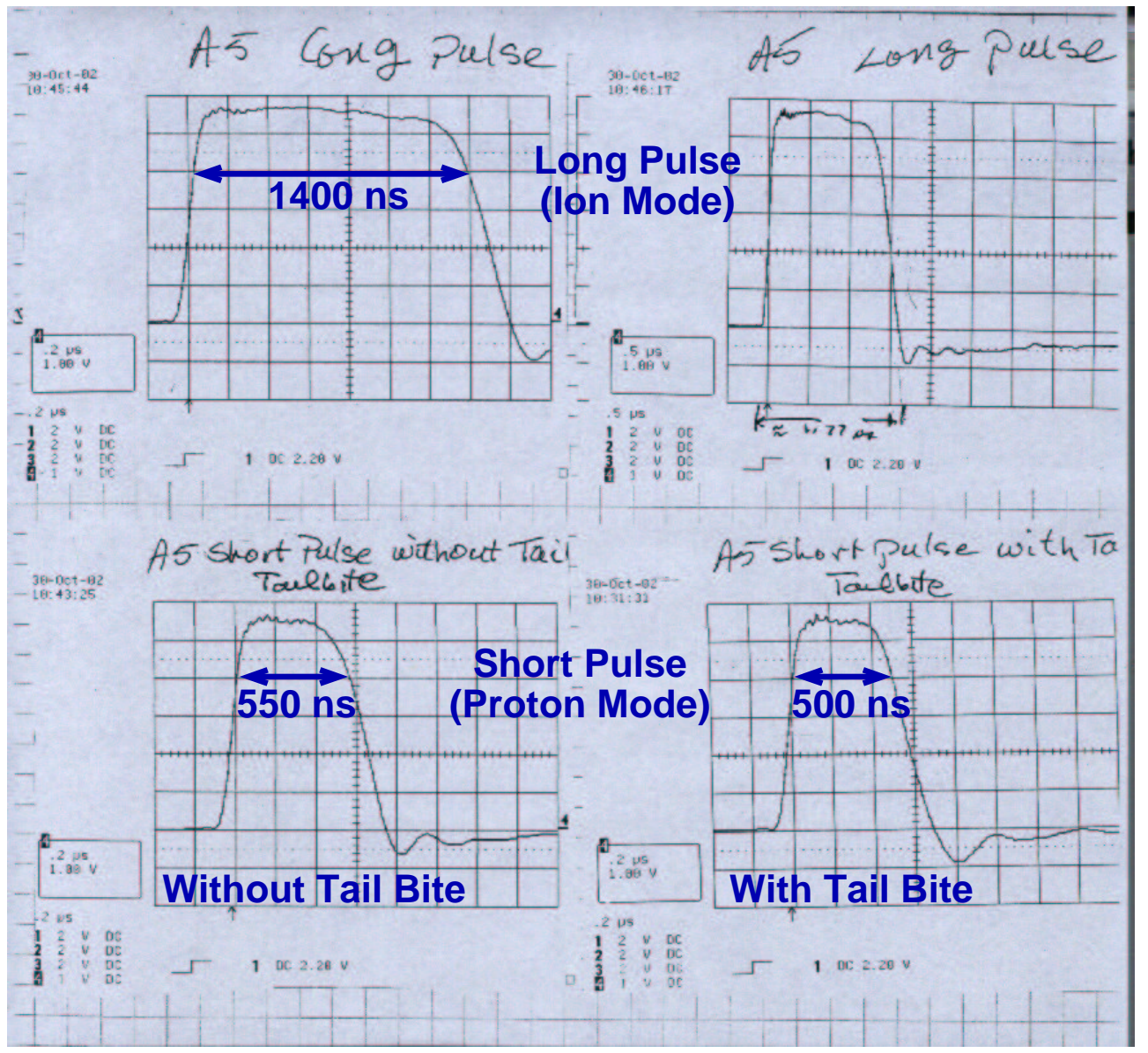

Figure 3: A5 kicker pulse widths in the ion and proton modes. The time per division is $200 \mathrm{~ns}$ for all traces except the upper right one which has a time per division of $500 \mathrm{~ns}$. 


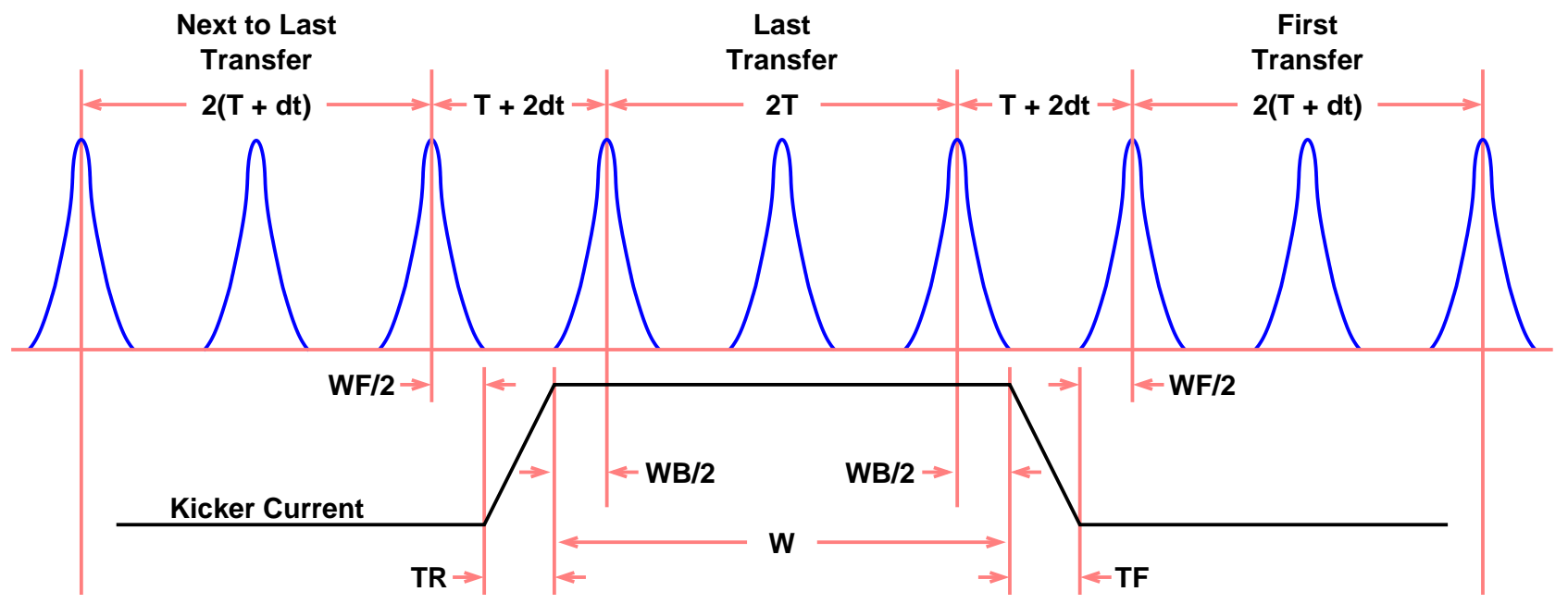

Figure 4: A5 Kicker timing for injection of $N=3$ bunches per transfer from Booster. Here $W=2 T+W_{B}$ and $T_{G}-W=2 T+4 d t-\left(W_{B}+W_{F}\right)$. For $T=260 \mathrm{~ns}, d t=5 \mathrm{~ns}, W_{B}=86 \mathrm{~ns}$, and $W_{F}=96 \mathrm{~ns}$, we have $W=606 \mathrm{~ns}$, $T_{G}=964 \mathrm{~ns}, T_{R}<179 \mathrm{~ns}$, and $T_{F}<179 \mathrm{~ns}$.

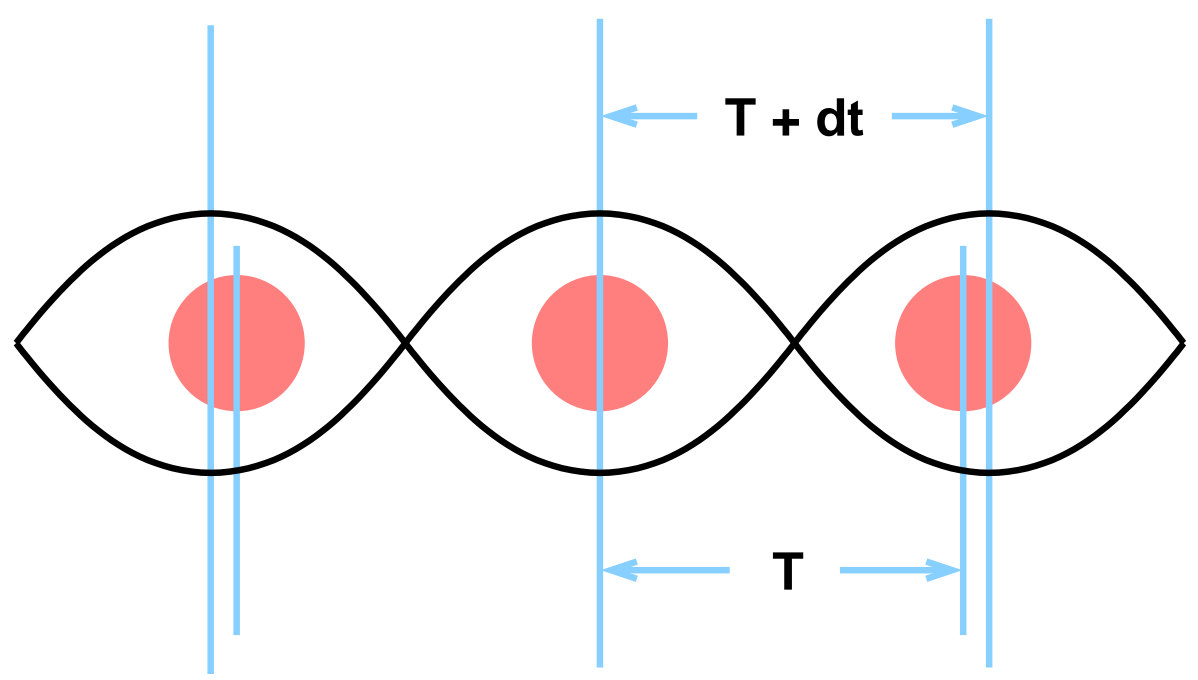

Figure 5: Phase mismatch between incoming bunches and RF buckets with three bunches per transfer. Here $T=260 \mathrm{~ns}$ and $d t=5 \mathrm{~ns}$. The displacement from center of bucket to center of bunch is $d t$ in the outer buckets. 

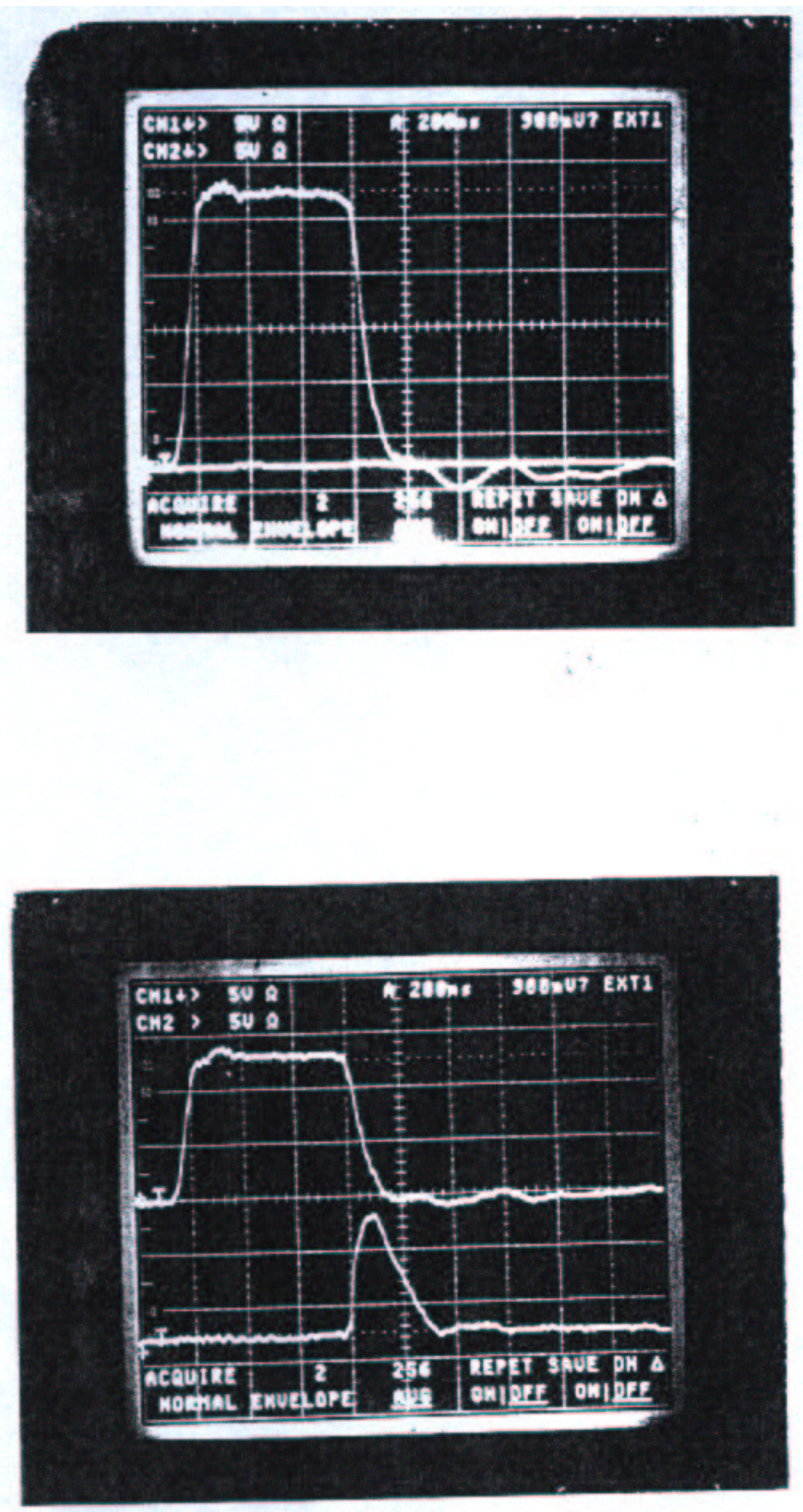

Figure 6: A5 kicker pulse in the original short pulse (proton) mode. The time per division is 200 ns. The bottom and top photos show the kicker pulse with and without the "tailbite" respectively. 


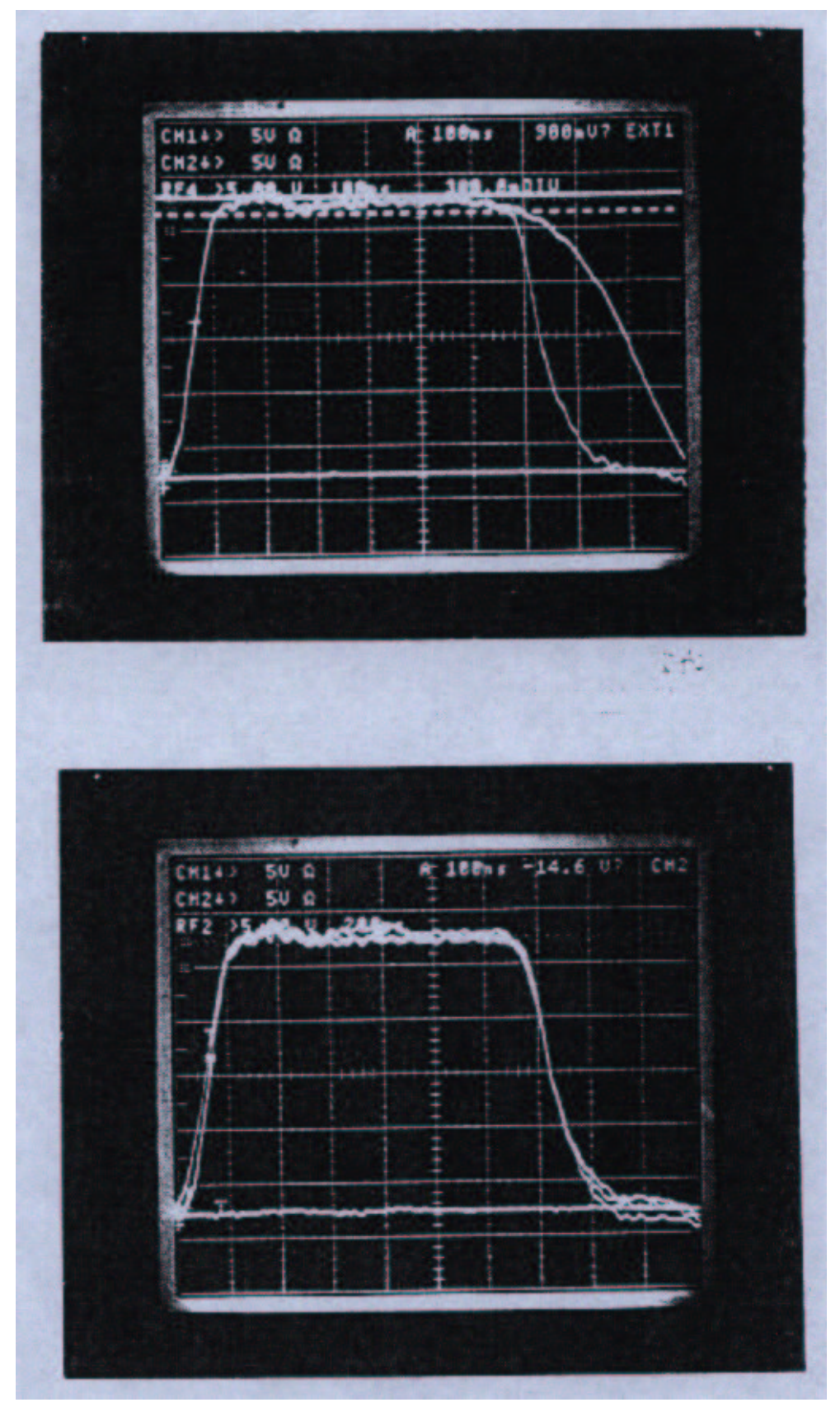

Figure 7: A5 kicker pulse in the original short pulse (proton) mode. Here the time per division is $100 \mathrm{~ns}$. The two traces in the top photo show the kicker pulse with and without the "tailbite". The bottom photo shows the pulses from the three modules of the kicker. 


\section{Acknowledgements}

Many thanks to J.M. Brennan for explaining his acceleration schemes, and to L.A. Ahrens for a critical reading of this note.

\section{References}

[1] L.A. Ahrens, et al, "Status and Recent Performance of the Accelerators that Serve as Gold Injector for RHIC", PAC2001, pp. 3184-3186.

[2] The notion of reducing the energy straggling by using foils with a more uniform surface thickness was introduced and championed by Peter Thieberger. Using a beam of protons from Tandem, he verified the effect for various foils. In particular, a silica foil showed promising results and this was used during part of the FY2003 RHIC run to strip gold ions in BTA from charge state 32 to 77 . Measurements by Christoph Montag, using turn-by-turn signals from the AGS wall monitor, showed that the silica foil reduced the energy straggling by a factor of two to three compared to the carbon foil used in the past. Here the technique of tomographic reconstruction of the longitudinal phase space was employed. This technique is described by C. Montag, et al, in Phys. Rev. Special Topics-Accelerators and Beams, Volume 5, 082801 (2002). 